Mon. Not. R. Astron. Soc. 000, 四 (2001) Printed 27 October $2018 \quad$ (MN LATEX style file v1.4)

\title{
The symbiotic star CH Cygni. I. Non-thermal bipolar jets
}

\author{
M. M. Crocker ${ }^{1}$, R. J. Davis ${ }^{1}$, S. P. S. Eyres², M. F. Bode ${ }^{3}$, \\ A. R. Taylor ${ }^{4}$, A. Skopal ${ }^{5}$ and H. T. Kenny ${ }^{6}$ \\ ${ }^{1}$ Jodrell Bank Observatory, University of Manchester, Macclesfield, Cheshire, SK11 9DL, UK. \\ ${ }^{2}$ Centre for Astrophysics, University of Central Lancashire, Preston, PR1 2HE, UK \\ ${ }^{3}$ Liverpool John Moores University, Twelve Quays House, Egerton Wharf, Birkenhead, CH41 1LD, UK \\ ${ }^{4}$ The Department of Physics and Astronomy, The University of Calgary, 2500 University Dr. N.W., Calgary, Alberta, T2N 1N4, Canada \\ ${ }^{5}$ Astronomical Institute, Slovak Academy of Sciences, 05960 Tatranská Lomnica, Slovakia \\ ${ }^{6}$ Department of Physics, Royal Military College of Canada, P.O. Box 17000, Stn Forces, Kingston, ON, K7K 7B4, Canada
}

Accepted ????. Received ????; in original form ????

\begin{abstract}
VLA surface brightness and spectral index maps of the evolving extended emission of the triple symbiotic star CH Cygni are presented. These are derived from observations at 4.8, 8.4 and $14 \mathrm{GHz}$ between 1985 and 1999. The maps are dominated by thermal emission around the central bright peak of the nebula, but we also find unambiguous non-thermal emission associated with the extended regions. Our observations confirm that this is a jet. The central region has been associated with the stellar components through HST imaging. If the jets are due to ejection events at outburst, expansion velocities are consistent with those from other measurement methods. We propose that the non-thermal emission is caused by material ejected in the bipolar jets interacting with the circumstellar wind envelope. The resulting shocks lead to local enhancements in the magnetic field from the compact component of the order $3 \mathrm{mG}$.
\end{abstract}

Key words: binaries: symbiotic - circumstellar matter - stars: imaging - stars: individual: CH Cygni - stars: late-type - radio continuum : stars

\section{INTRODUCTION}

Symbiotic stars occupy an extreme and relatively poorly understood region of the binary classification scheme. The name was coined by Merril (1941) to describe stars which appeared to have a combination spectra: that of high excitation lines usually associated with a hot, ionized nebula superimposed on a cool continuum with prominent absorption features consistent with a late-type star. At present they are understood to be interacting binaries (with orbital periods of a few to tens of years) consisting of a cool giant losing material mostly via the stellar wind and a hot, luminous compact object which ionises a portion of the cool component wind (Seaquist et al., 1984, hereafter the STB model). Such a state of affairs represents the so-called quiescent phase, which can be interrupted by periods of activity. The active phases start with an eruption of the hot star, an event indicated photometrically by an increase of the star's brightness by 2-6 mag, and/or spectroscopically by high velocity broad emission features from the central star. Both radio and HST imaging can directly resolve the remnants of such dramatic events (Hack \& Parsece, 1993; Eyres et al. 1995; Kenny et al. 1996; Richards et al., 1999; Eyres et al., 2001; Eyres et al. in preparation, hereafter Paper II).

The symbiotic star CHCygni displays particularly complex behaviour. Optical spectroscopic studies by Mikołajewski et al (1987) showed that the orbits of the stars within the system are likely to be coplanar and eclipsing, with eclipses separated by around 5700 days. Later studies (Mikołajewski et al, 1990 and references therein) confirmed this period.

Further spectral studies of the system (Hinkle et al. 1993) suggested that CH Cyg is probably a triple-star system consisting of an inner 756-day period binary which is orbited by an unseen G-K dwarf on a 5300 day orbit. Further photometric studies (Skopal et al., 1996) discovered short, 756 day, period eclipses which show that all three stars in the system are likely to be in coplanar orbits.

The activity of $\mathrm{CHCyg}$ is seen best in the $U$ lightcurve (Skopal, 1998), which indicates a brightening from $U \sim 11-12$ to 5 (Fig. 1). The drop in the optical magnitude of CH Cygni at the times of the long-period eclipses have been marked on this figure. Each outburst was accompanied by high velocity broad emission features consistent with mass outflows. During 1984-85 the material was ejected at $\sim 600-2500 \mathrm{~km} \mathrm{~s}^{-1}$ (Mikołajewski \& Tomov 1986), whilst the 1992-95 active phase was characterized by sporadic and in part bipolar outflow at $\sim 1000-1600 \mathrm{~km} \mathrm{~s}^{-1}$ (Leedjärv \& Mikołajewski, 1996; Skopal et al. 1996; Ijima 1996) and, fi- 


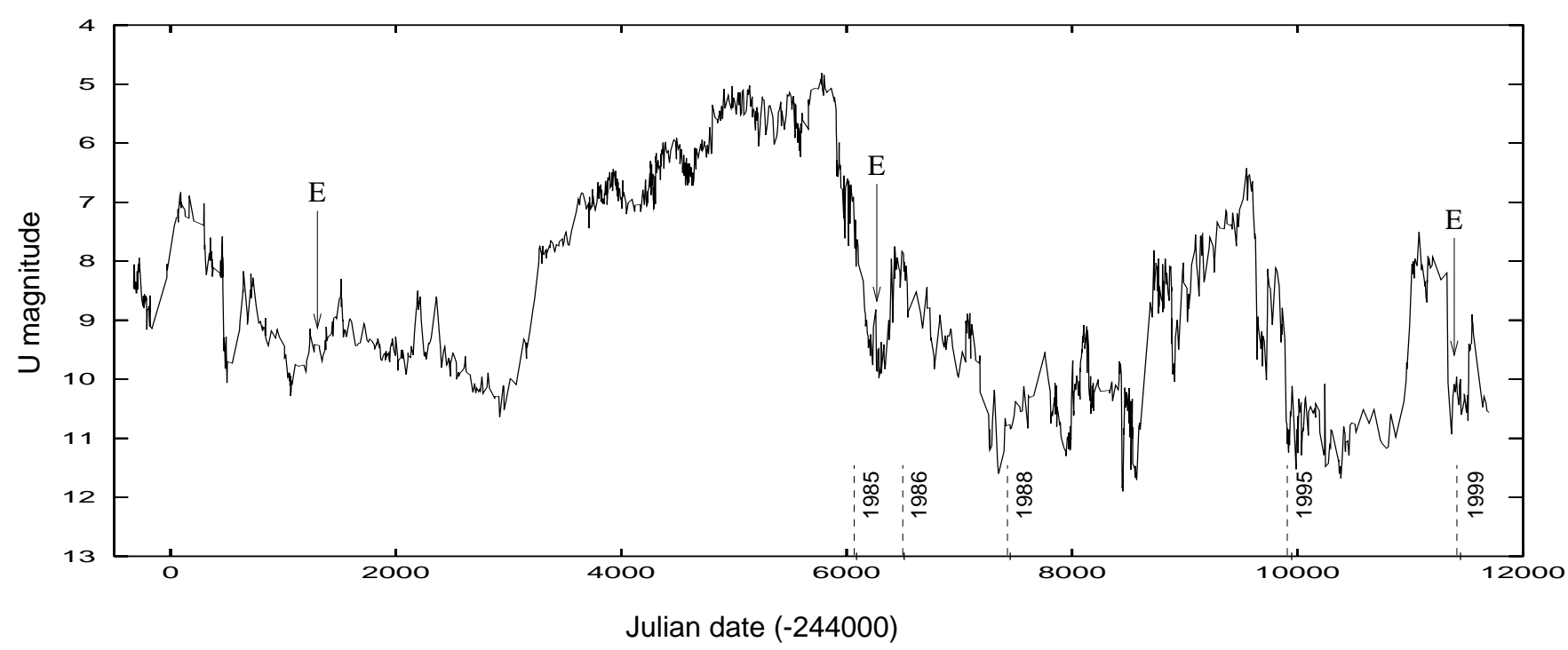

Figure 1. The $U$ light-curve for CH Cyg covering the period $1967-2000$. The eclipses in the long, 14.5-year, period orbit are marked by $\mathbf{E}$. The timings of our VLA observations pertinent to this paper are denoted by dashed bars.

Table 1. VLA Observations of CH Cygni.

\begin{tabular}{lllllll}
\hline Date & $\begin{array}{l}\text { Band 1 } \\
(\mathrm{GHz})\end{array}$ & $\begin{array}{l}\text { Band 2 } \\
(\mathrm{GHz})\end{array}$ & $\begin{array}{l}\text { Time on Source } \\
\text { at Band 1(s) }\end{array}$ & $\begin{array}{l}\text { Map 1noise } \\
\left(\mathrm{mJy} \mathrm{beam}^{-1}\right)\end{array}$ & $\begin{array}{l}\text { Time on Source } \\
\text { at Band 2 }(\mathrm{s})\end{array}$ & $\begin{array}{l}\text { Map 2 noise } \\
\left(\mathrm{mJy} \mathrm{beam}^{-1}\right)\end{array}$ \\
\hline 1986 Mar 20 & 4.8 & 15 & 2290 & 0.053 & 9930 & 0.083 \\
1988 Oct 16 & 4.8 & 15 & 2600 & 0.032 & 5220 & 0.073 \\
1995 Aug 20 & 4.8 & 8.5 & 2550 & 0.021 & 5620 & 0.063 \\
1999 Sep 26 & 4.8 & 8.5 & 3210 & 0.077 & 2400 & 0.133 \\
\hline
\end{tabular}

${ }^{a}$ referred to as $\sigma_{\text {rms }}$

nally, during the recent, 1998-2000, outburst mass outflows at about $1000 \mathrm{~km} \mathrm{~s}^{-1}$ were observed (Paper II). The outflows may be correlated with a significant increase of the radio emission and the radio light curves during these periods correlate well with the optical ones (Kenny et al. 1996). The 1984 mass ejection has been linked to emergence of bipolar emission which could be from either an expanding torus or collimated jets (Taylor et al. 1986). Later radio observations revealed a complex morphology, although still exhibiting a bipolar structure (Kenny et al. 1996).

In this paper we present radio surface brightness and spectral index maps taken from 15 years of observations of CH Cygni by the Very Large Array.

\section{OBSERVATIONS}

$\mathrm{CH}$ Cygni has been observed in the highest resolution "A" configuration several times with the VLA since 1984 and multiple frequency observations were made on many of these occasions, four of which are detailed in Table 1 .

Data reduction was carried out according to standard procedures using the AIPS software package (Greisen, 1999). The flux density of the phase reference source was determined, and the data calibrated to correct phase and am- plitude instabilities. These corrections were applied to the observations of CH Cygni and a map produced by deconvolving the instrumental response (the so-called "dirty beam") from the map using the well-established CLEAN algorithm (Högbom, 1974). Due to the low radio flux density of the source no self-calibration was possible. The maps were convolved with a beam size of $0.5 \times 0.5$ arcsec at all observing bands. This size was the mean of the the major and minor axes of the beam at $5 \mathrm{GHz}$, in order that comparison of the size and shape of features at different epochs would be meaningful.

Observations of the same object at different frequencies have a different $u, v$ plane coverage. This leads to features of different spatial scales being picked out at the different frequencies, causing problems when taking a ratio between two maps. In order to avoid this, only the $u, v$ range covered by both sets of data was used to create the maps. This has the undesirable effect of decreasing the signal-to-noise ratio but does mean that the maps can be compared. The AIPS task "comb" was then used to produce a third map (at a resolution of 0.17 arcseconds per pixel) which traces the pixel-by-pixel spectral index variation. We use the convention that the radio flux at frequency $\nu, S_{\nu} \propto \nu^{\alpha}$ so that 


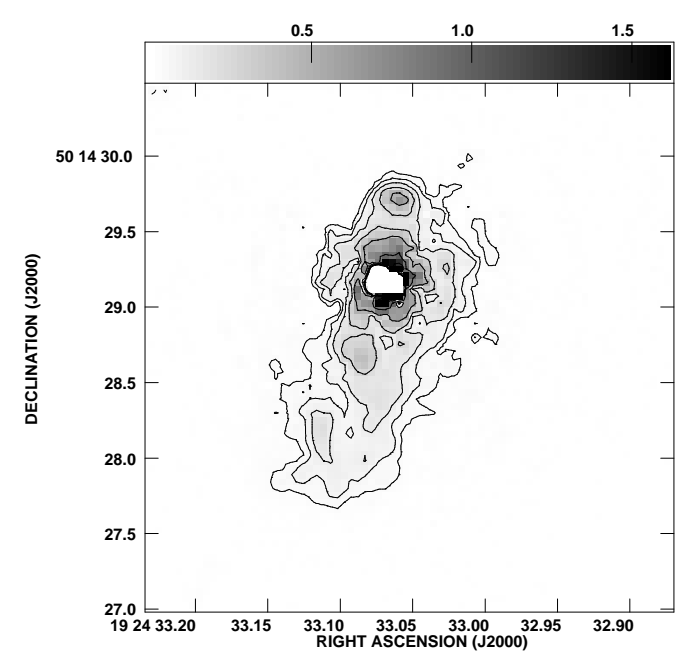

Figure 2. HST WFPC2 image of CH Cygni from 1999 August 13. The point spread function of the central star has been subtracted from this image for clarity.

a positive spectral index $(\alpha)$ represents an increase in flux with frequency.

Hubble Space Telescope imaging was used to place the stellar components on the radio map. The observations were made as part of a GO programme 8330 on symbiotic stars. Three orbits were allocated to $\mathrm{CH}$ Cygni. A WFPC2 image (Fig. 2) taken through the F502N filter (Biretta 1996) on 1999 August 13 was aligned to the VLA map of 1999 September. The best agreement was achieved when the radio peak coincided with the star in the optical domain (this is discussed in more detail in Paper II).

\section{RESULTS}

The radio brightness and spectral index maps are shown in Fig. 3. The brightness maps are dominated by a central bright peak surrounded by an extended region. The morphology of these extensions varies from one epoch to the next. In particular, after the initial bipolar extension observed in 1986 the extension appears to be more pronounced on the SE side of the central peak. In addition to this the position angle of the extension has changed (Crocker et al, in preparation).

In each spectral index map, there is a wide range of indices visible. In particular, the 1986 map (Fig. B, top) shows a distinct trend with positive $\alpha$ in the central region and negative $\alpha$ in the outlying areas. This strongly suggests the existence of thermal emission around the stellar components with extended non-thermal emission from the well-resolved knots.

Cross-sections were taken through the peak of each spectral index map, along the axis of maximum elongation in the $5 \mathrm{GHz}$ map. In order to reduce the uncertainties, a section was taken on each side of the initial one, giving three parallel sets of data. At each point along the slice, the three pixels were combined to find a weighted mean and then used to create the final cross-section. The weighted mean crosssections are shown in Fig. 4, where the positions are given relative to the central bright peak in the $8.5 \mathrm{GHz}$ or $15 \mathrm{GHz}$ radio maps, whichever was available. A feature common to all the epochs is a central peak, accompanied by wings of lower spectral index.

The first plot, from 1986, has a peak value of $\alpha=0.42 \pm$ 0.03 which is consistent with that expected from a constant outflow ionized wind, assuming a variation in density of $\rho \propto$ $r^{-\beta}$, with $\beta=1.8$. The decrease in $\alpha$ with distance from the centre favours a geometry which is optically thick along lines of sight that pass through a dense core. In the extended regions, the mean value of $\alpha$ is $-0.23 \pm 0.03$.

By 1988, the spectral index of the central region had increased to $\alpha=1.02 \pm 0.04$ as if $\beta>2$, which can be attributed to the photoionised nebula dominating over the wind. Such a value is predicted by the STB model for lower ionisation parameters. No extended emission is seen at $15 \mathrm{GHz}$. This observation was made at a time when the optical activity was very low (Fig. 11) and no outburst had been observed for some time.

The 1995 cross-section has a peak of similar width to the other epochs in conjunction with bright thermal emission $(\alpha>0.5)$ in the extension seen to the SE of the central source in the $8.5 \mathrm{GHz}$ map in Fig. 3. This feature may be a result of local condensation in the mass flow.

In 1999, almost the entire emitting region has a negative spectral index, with a minimum of $\alpha=-0.75 \pm 0.07$. This observation was made during what may be an eclipse of the inner symbiotic region by the outer giant (Skopal 1997). This may have reduced the relative intensity of the thermal emission, suggesting that the thermal emission region was very compact at this time, i.e. similar in extent to the angular diameter of the outer red giant.

\section{DISCUSSION}

\subsection{The geometry of the extended emission}

The extensions detected in the radio surface brightness maps are more likely to be collimated jets rather than a torus. A torus is unlikely to appear so obviously one-sided, following an initial bipolar morphology. The non-thermal emission is suggestive of high velocity flow, commonly associated with jets but not with a circumbinary torus.

It may be that the extended emission is due to a large nebula, with a radius of order 100AU, which has been photoionised between 1985 and 1986. From the STB model, for suitable parameters $\left(T_{\mathrm{H}}=100,000 \mathrm{~K}, \dot{M}=\right.$ $1.9 \times 10^{-6} M_{\odot} \mathrm{yr}^{-1}$ (Skopal et al., 1996), $V_{\text {wind }}=20 \mathrm{~km} \mathrm{~s}^{-1}$ (Vogel et al., 1994)) it is clear that the ionised cavity due to the hot component will be density bounded and much smaller than the binary separation.

\subsection{Expansion velocity}

The Hipparcos distance to CH Cygni is $268 \pm 66$ pc (Viotti et al. 1998), meaning that the maximum extent of the $3 \sigma_{\text {rms }}$ contour of radio emission at $15 \mathrm{GHz}$ in 1986 is $\sim 500 \mathrm{AU}$. The most likely origin of of extended non-thermal emission is synchrotron emission from relativistic electrons in a magnetic field.

If CH Cygni follows the model proposed for HM Sagittae by Eyres et al. (1995), then what is being observed are regions of shocked material, caused when high velocity ejecta 


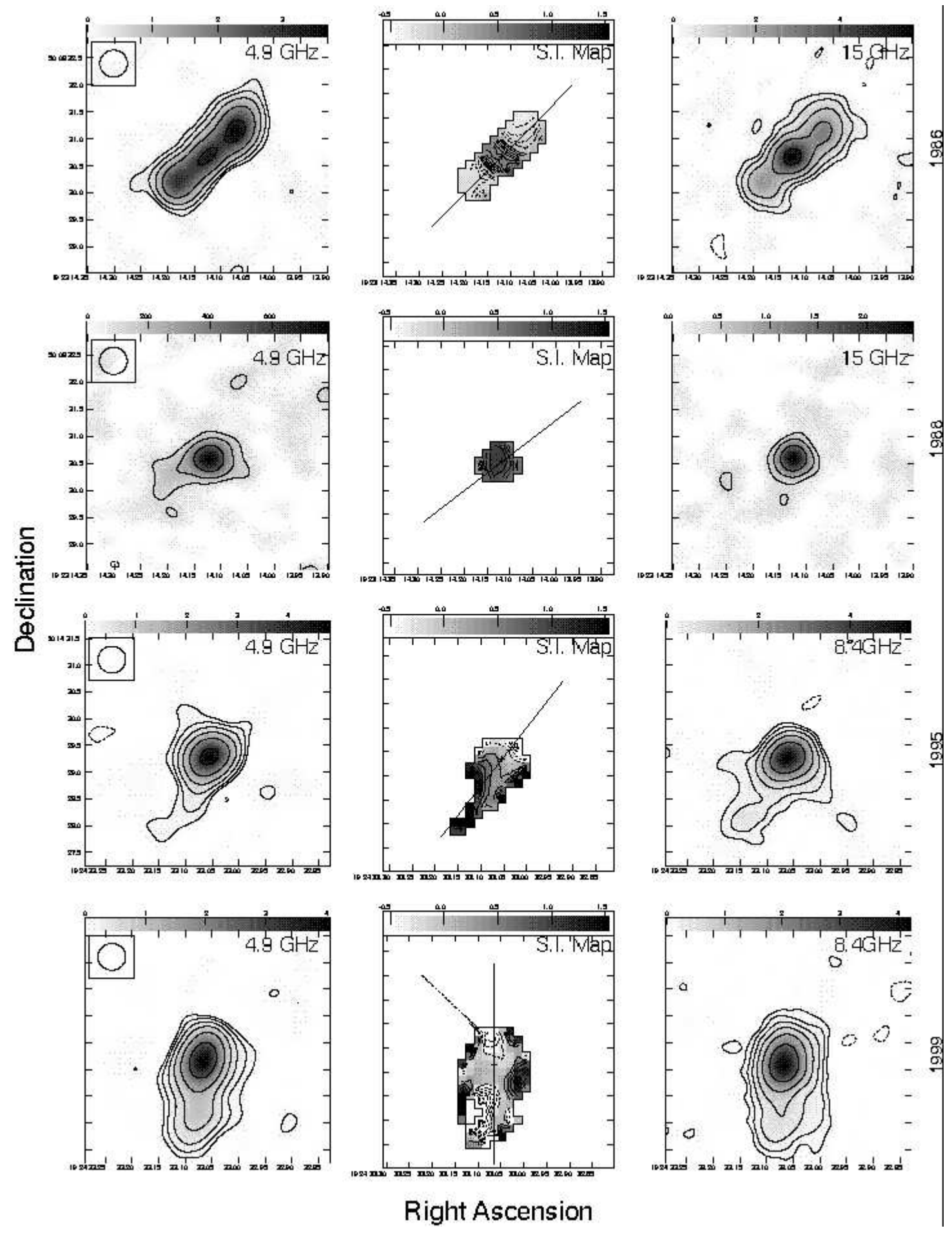

Figure 3. VLA spectral index maps (centre) created from $4.8 \mathrm{GHZ}$ (left) and $8.5 \mathrm{GHz}$ or $15 \mathrm{GHz}$ (right) maps from from all epochs. The lowest contour line on the brightness maps is $\sigma_{\mathrm{rms}} \times-3,3,6,12,24,48,96$ where present. The values of $\sigma_{\mathrm{rms}}$ for each map are given in Table 11. The white to black range on the spectral index map is $-0.5 \leqslant \alpha \leqslant+1.5$ and the contours represent changes in $\alpha$ of 0.1 . 


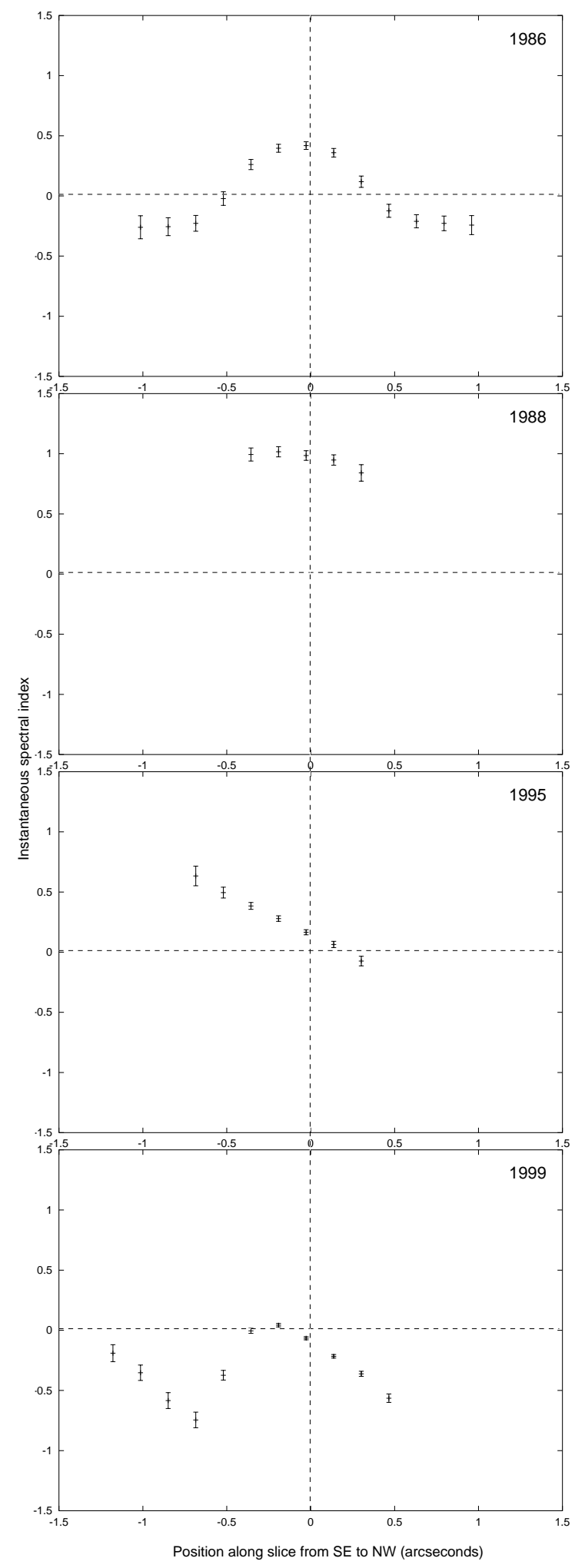

Figure 4. Plots of instantaneous spectral index variations along the lines marked out in Fig. 3, from SE to NW, for each of the maps, relative to the bright peak in the higher frequency radio brightness map. expand into the relatively dense circumtrinary envelope created by the winds of the giant stars in the system.

Evidence for this scenario comes from infrared spectroscopy (Taranova \& Yudin, 1988), carried out around the period of the end of the optical outburst in 1983-1987, showing changes in the circumtrinary dust envelope. They suggested that the dust was disrupted at the time of the radio jet emergence, probably as it was swept up by the expanding matter.

The positions of the NW and SE extended knots were measured on the $198615 \mathrm{GHz}$ radio map. The angular separations between the peak in each knot and the central bright peak were

$$
\Delta \theta_{\mathrm{NW}}=0.70 \pm 0.01 \operatorname{arcsec}
$$

and

$$
\Delta \theta_{\mathrm{SE}}=0.71 \pm 0.05 \operatorname{arcsec}
$$

for the NW and SE knots respectively. The greater uncertainty in the SE position is due to the fact that the SE component has lower surface brightness than the the NW one. In a 1985 VLA $15 \mathrm{GHz}$ map (Fig. 月), these two knots were not visible, and hence the material must have been ejected within 422 days before the 1986 observation. This places a lower limit on the expansion velocity. Assuming a distance to $\mathrm{CH}$ Cyg of $268 \pm 66$ pc the velocities are found to be

$$
V_{\mathrm{NW}} \geqslant 1210 \pm 300 \mathrm{~km} \mathrm{~s}^{-1}
$$

for the NW component and

$$
V_{\mathrm{SE}} \geqslant 1230 \pm 320 \mathrm{~km} \mathrm{~s}^{-1}
$$

for the SE component, with the uncertainties dominated by the uncertainty on the Hipparcos distance. These results correspond well with those derived previously, in particular with the expansion velocity of $1400 \mathrm{~km} \mathrm{~s}^{-1}$ derived from two earlier maps (Taylor et al. 1986) that were separated by only 75 days.

\subsection{Magnetic field in 1986}

The upper limit of the surface magnetic field of a typical white dwarf is around 10G (Bond and Chanmugam, 1982). Assuming a dipole geometry, this will fall off as $B_{\mathrm{WD}} \propto$ $r^{-3}$. For a stellar radius of $0.01 \mathrm{R}_{\odot}$ the upper limit on the magnetic field at $500 \mathrm{AU}$ is $80 \mu \mathrm{G}$, which is far too low to give efficient synchrotron emission.

The speed of the unshocked pre-existing cool wind is likely to be much less than the jet speed, as a typical cool giant wind has a velocity of the order of $20-40 \mathrm{~km} \mathrm{~s}^{-1}$ (Vogel et al. 1994). The non-thermal emission is then caused by particle acceleration at the resulting bow shock, as the local magnetic field is compressed and enhanced and electrons are accelerated to velocities close to $c$. In such an environment, the local azimuthal field, $H_{\phi}$ is defined by Equation 42 of Bode \& Kahn (1985) the density of the shock, the temperature of the giant wind (assumed to be constant at $\sim 10^{4} \mathrm{~K}$ ) and the mean particle mass which is taken to be the proton 


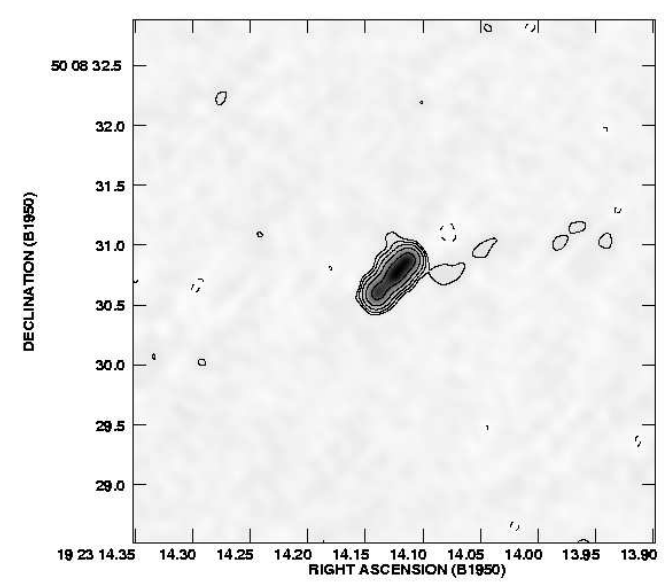

Figure 5. VLA $15 \mathrm{GHz}$ map from 1985 January 22. The contours are as in Fig 3 and the rms noise level is $0.19 \mathrm{mJy}$ per beam.

mass. The shock is assumed to be strong and totally ionised, so that the shock density is equal to 4 times the density of the unshocked wind at this distance from the star. It is assumed that the disruptions caused to the giant wind by the hot component prior to mass ejection are negligible at this distance so that a uniform, spherical radial wind exists.

The denisty of the wind is proportional to the mass loss rate of the giant, $\dot{M}=1.9 \times 10^{-6} \mathrm{M}_{\odot} \mathrm{yr}^{-1}$ (Skopal, 1996). If the unshocked wind speed, $v_{w}$ is taken to be the escape velocity of the red giant, assuming $M_{\text {giant }}=0.93 M_{\odot}$ and $R_{\text {giant }}=123 R_{\odot}\left(\right.$ Skopal 1997) then $v_{w}=53.8 \mathrm{~km} \mathrm{~s}^{-1}$ and $H_{\phi} \simeq 2.4 \mathrm{mG}$.

Another magnetic field estimate can be derived from the technique used in studying high energy extragalactic sources. The total energy of a radio source is split between that of the electrons, the protons and the magnetic field. At equipartition, the magnetic energy is approximately equal to the particle energy (Pachoczyk, 1970), and the ratio of energy carried by protons to that carried by electrons is $K$. For a radio source of known angular dimensions and depth, assuming an ellipsoidal geometry, the magnetic field under equipartition, $H_{E}$, can be found using the method described by Miley (1980).

To make use of this model, one needs to determine several parameters that describe the region: filling factor, $\eta$; the angle between the magnetic field and the line of sight, $\chi$; the flux density of the emitting region, $S_{\nu}(J y)$, at frequency $\nu$ $(\mathrm{GHz})$ and the maximum and minimum frequencies of radio emission $\left(\nu_{\max }\right.$ and $\nu_{\min }$ repectively, in $\mathrm{GHz}$ ) from the source spectrum.

The filling factor would be more useful in extragalactic work when it would be expected that there would be unresolved blobs of emitting material. Since the measured sizes of the knots in the extended emission are of a similar angular size to the beam, $\eta=1$. The angle $\chi$ is important due to the fact that the radiation observed is beamed from electrons moving towards the observer. The fact that eclipses are seen in the system means that the orbital plane of the stars is observed edge-on. Bipolar jets are believed to be aligned with the rotation axes of their parent star (perpendicular to the orbital plane) so the jets of $\mathrm{CH}$ Cygni are believed to lie almost in the plane of the sky (Crocker et al., in preparation), $\phi \simeq 90^{\circ}$. The cutoff frequencies of the radio emission, $\nu_{\min }$ and $\nu_{\max }$ are taken to be $0.01 \mathrm{GHz}$ and $100 \mathrm{GHz}$ respectively (Miley, 1980). The value of $K$ is the main unknown in this equation as the exact details of the mechanism leading to synchrotron emission are not known. A value of $K=100$ would be appropriate for the electrons being produced following collisions in a circumstellar medium $(\mathrm{Pa}-$ cholczyk 1970), but a value of $K=1$ is more consistent with the minimum energy requirement (Miley 1980). However, since $H$ only depends upon $K^{\frac{2}{7}}$, having $K 100$ times larger would only increase $H_{E}$ by a factor of 3 .

In order to determine the size of the jet bullets, two dimensional gaussians were fitted to the emitting regions in the NW and SE jets. The depth was then found by assuming that the regions exhibited cylindrical symmetry along the axis of ejection. For the NW jet, $\theta_{1}=0.39 \operatorname{arcsec}, \theta_{2}=0.34$ arcsec and $d=91 \mathrm{AU}$. The SE jet had dimensions of $\theta_{1}=$ 0.37 arcsec, $\theta_{2}=0.35$ arcsec and $d=94$ AU. Using the mean value of the spectral index in the jets of $\alpha=-0.23 \pm 0.03$,

$$
H_{E}=3.8 \mathrm{mG}
$$

for the NW jet and

$$
H_{E}=3.6 \mathrm{mG}
$$

in the SE. These values are comparable to that found above from the physics of the shock, so there is sufficient magnetic field in the enhanced regions for synchrotron radiation.

\subsection{Lifetimes}

Since the total luminosity of this region at all frequencies is not known, determining the lifetime of the emission due to all radiative energy losses is impossible. In addition to this, synchrotron loss processes may be dominant. Under the influence of a magnetic field $H$, an electron rotates at the gyrofrequency (Rybicki \& Lightman, 1979) and the frequency of radiation, $\nu$, is a factor of $\gamma^{3}$ higher than the gyrofrequency, so manipulating Equation 6.4 of Rybicki \& Lightman (1979) gives $\gamma=1148$. An electron with this Lorentz factor has energy $\gamma m_{e} c^{2}$ and radiates with power $P$ so that the lifetime of the synchrotron radiation is

$$
\tau=\frac{9 m_{e}^{3} c^{5}}{4 \gamma e^{4} H^{2}}
$$

and with the values of $\gamma$ and $H=H_{\phi}$ from above, $\tau=$ $4.7 \times 10^{10}$ seconds, corresponding to just under 1500 years. Such a timescale would imply that synchrotron radiation would be very long-lived in the circumstellar material, assuming that the magnetic field compression is sustained by continuous matter injection. When there is no matter injection the magnetic field can expand adiabatically and the synchrotron emission will fade within years. No non-thermal emission is seen in 1988. If indeed there is no synchrotron emission at this epoch, some other loss-mechanism must be occurring. The optical light curve of figure 1 shows 1988 to be a particularly quiet period, over two years after the last major outburst. It is therefore likely that there is no matter being ejected into the circumstellar wind, so that no new 
density enhancements and magnetic field compressions are being formed.

Supporting evidence for this scenario comes from optical spectral observations. During this epoch, the blue continuum was very faint and the Balmer lines were not present (Bode et al. 1991), strongly implying that there were also no fast winds or jets at this time. Hence one would not expect to detect shock phenomena resulting from penetration of high-velocity material into circumbinary material and, by this model, no non-thermal emission from recently ejected material.

\subsection{Comparison with other symbiotic stars}

Previously, unambiguous non-thermal emission in symbiotic stars had only been seen in HM Sge (Richards et al. 1999). Spatially resolved non-thermal emission is very rare in any stellar source, and is only seen regularly in very high-energy systems such as micro-quasars. A potential non-thermal feature in R Aqr was subject to such large errors $\left(\sigma_{\alpha} \sim 1\right)$ that its status could not be confirmed (Dougherty et al. 1995). Other symbiotic stars are believed to exhibit entirely thermal radiation. $\mathrm{CH}$ Cyg displays the strongest evidence yet of non-thermal emission due to jet-wind interaction. $\mathrm{CH} \mathrm{Cyg}$ and HM Sge share the property of having undergone outbursts in relatively recent history. The above arguments suggest that non-thermal emission will decrease rapidly with time after the outburst if the field strength and high energy particles are not continuously replenished as discussed initially in Richards et al. (1999). Since the 1984-86 ejections in CH Cygni two outbursts have been observed which may have injected further high velocity material into the circumstellar environment and hence given rise to the non-thermal emission seen in the 1995 and 1999 observations.

\section{CONCLUSION}

There is definite evidence of non-thermal emission in the ejecta from $\mathrm{CH}$ Cygni. This, together with a morphology consistent with high velocity jets, supports the scenario of Taylor et al. (1986), rather than one where the extension is a result of an expanding circumstellar torus. The proposed mechanism for the creation of the non-thermal emission involves material ejected in high velocity bullets interacting with a previously expelled lower velocity stellar wind. The ejected matter creates shocks in the circumstellar medium, leading to locally enhanced magnetic field density, particle acceleration and synchrotron emission. We suggest that the presence of non-thermal emission in symbiotic stars may be an indicator of outbursts associated with significant ejecta.

\section{ACKNOWLEDGMENTS}

MC is supported by a grant from the Particle Physics and Astronomy Research Council (PPARC). The contribution of AS was supported by a grant of the Slovak Academy of Science, number 5038/2000. The VLA is operated by the National Science Foundation operated under cooperative agreement with Associated Universities, Inc.

\section{REFERENCES}

Biretta J., 1996, WFPC2 Handbook, Version 4.0. STScI

Bode M. F., Kahn F. D., 1985, MNRAS, 217, 205

Bode M. F., Roberts J. A., Ivison R. J., Skopal A., 1991, MNRAS, 253, 80

Bond H. E., Chanmugam G., 1982, Advances in Ultraviolet Astronomy, 530

Dougherty S. M., Bode M. F., Lloyd H. M., Davis R. J., Eyres S. P., 1995, MNRAS, , 843

Eyres S. P., Kenny H. T., Cohen R. J., Lloyd H. M., Dougherty S. M., Davis R. J., Bode M. F., 1995, MRNAS, 274,317

Eyres S. P. S., Bode M. F., Taylor A. R., Crocker M. M., Davis R. J., 2001, ApJ, 551, in press

Eyres S. P., Bode M. F., Taylor A. R., Crocker M. M., Davis R. J., 2000, ApJ, submitted

Greisen E., 1999, The AIPS Cookbook, NRAO.

Hack. W. J.., Parsece F., 1993, PASP, 105, 1273

Hinkle K. H., Fekel F. C., Johnson D. S., G. S. W. W., 1993, AJ, 105,1074

Ḧogbom J. A., 1974, A\&AS, 15, 417

Iijima T., 1996, Astro. Lett. and Communications, 34, 327

Iijima T., 1998, in Koyama K., ed, The Hot Universe. IAU, p. 219

Kenny H. T., Taylor A. R., Frei B. D., Bang M., Bode M. F., Davis R. J., Eyres S. P. S., Ivison R. J., Seaquist E. R., Skopal A., 1996, ASP Conference Series, 93, 197

Kenyon S. J., 1986, The Symbiotic Stars, CUP.

Leedjärv L., Mikołajewski M., 1996, A\& A, 300, 189

Merril P. W., 1941, PAAS, 10, 168

Mikołajewski M., Tomov T., 1986, MNRAS, 219P, 13

Mikołajewski M., Mikołajewska J., Tomov T., 1987, Ap\& SS, 131, 733

Mikołajewski M., Mikołajewska J., Khudyakova T. N., 1990, A\& A, 235, 219

Miley G., 1980, Ann. Rev. Astron. Astrophys., 18, 165

Pacholczyk A. G., 1970, Radio Astrophysics. W. H. Freeman and Company

Richards A. M. S., Bode M. F., Eyres S. P. S., Kenny H. T., Davis R. J., Watson S. K., 1999, MNRAS, 305, 380

Rybicki G. B., Lightman A. P., 1979, Radiative Processes in Astrophysics. John Wiley and Sons

Seaquist E. R., Taylor, A. R., Button, S., 1984, ApJ, 284, 202

Skopal A., Bode M. F.,Lloyd H. M., Tamura S., 1996, MNRAS, 282,327

Skopal A., Pribulla T., Wolf M., Shugarov S. Y., Jones A., 2000, Coska, 30, 29

Skopal A., 1997, in Mikołajewska J., ed, Physical processes in symbiotic binaries. Copernicus Foundation for Polish Astronomy, p. 99

Skopal A, Bode M. F., Lloyd M. H., Drechsel H, 1998, A\& A, 331,179

Tananova O. G., Yudin B. F., 1988, Ap\&SS, 146, 33

Taylor A. R., Seaquist E. R., Mattei J. A., 1986, Nature, 319, 38

Viotti R., Badiali M., Cardini D., A. E., T. I. 1997 Hipparcos, p. 405, ESA, 1998

Vogel M., Nussbaumer H., 1994, A\&A, 284, 145

Watson S. K., Eyres S. P. S., Davis R. J., Bode M. F., Richards A. M. S., Kenny H. T., 2000, MNRAS, 311, 449

Wright A. E., Barlow M. F. S., 1975, MNRAS, 170, 41 